\title{
The Physiological Effects of a Walking to Music Intervention in Adults with Intermediate Hyperglycemia
}

\author{
Maria Faulkner1, Marie Murphy², Gareth Davison², David Rowe ${ }^{3}$, Allan Hewitt ${ }^{3}$, Alan Nevill ${ }^{4}$, \\ Ellie Duly5, Tom Trinick5, Andrea M. McNeilly² \\ ${ }^{1}$ Letterkenny Institute of Technology, Donegal, Ireland \\ ${ }^{2}$ Sport and Exercise Sciences Research Institute, Ulster University, Newtownabbey, Northern Ireland \\ ${ }^{3}$ School of Psychological Sciences and Health, University of Strathclyde, Glasgow, UK \\ ${ }^{4}$ Faculty of Education Health \& Wellbeing, University of Wolverhampton, Wolverhampton, UK \\ ${ }^{5}$ Clinical Biochemistry Department, Ulster Hospital, South Eastern Health Trust, Belfast, Northern Ireland \\ Email: a.mcneilly@ulster.ac.uk
}

How to cite this paper: Faulkner, M., Murphy, M., Davison, G., Rowe, D., Hewitt, A., Nevill, A., Duly, E., Trinick, T. and McNeilly, A.M. (2021) The Physiological Effects of a Walking to Music Intervention in Adults with Intermediate Hyperglycemia. Open Journal of Endocrine and Metabolic Diseases, 11, 43-61.

https://doi.org/10.4236/ojemd.2021.111004

Received: April 27, 2020

Accepted: January 24, 2021

Published: January 27, 2021

Copyright $\odot 2021$ by author(s) and Scientific Research Publishing Inc. This work is licensed under the Creative Commons Attribution International License (CC BY 4.0).

http://creativecommons.org/licenses/by/4.0/

(c) (i) Open Access

\begin{abstract}
Purpose: Overweight individuals are at risk of intermediate hyperglycemia (IHG) [1]. Increasing physical activity (PA) in IHG is one method to reduce the risk of glucometabolic and cardiovascular (CV) complications [2]. This study investigates walking to music as a modality to increase moderate intensity PA and regulate glucometabolic disturbances. Patients and Methods: Participants were randomized to usual care (UC) or intervention group (IG) who completed a 6 month walking to music program. Physiological assessments for a range of variables (DEXA, flow mediated dilatation (FMD) and glucometabolic biomarkers) were completed at baseline, 4, 6 and 9 months (follow-up). Results: For IG group, walking compliance decreased with time however $71.4 \%, 79.5 \%$ and $73 \%$ of walking completed was moderate intensity at 4, 6 and 9 months. At 6 months IG FMD was significantly lower than UC and $\mathrm{HbA}_{1 \mathrm{C}}$ was $5 \%$ lower. Other physiological markers were not altered. Conclusion: Walking to music may be a novel method to encourage moderate intensity PA. However, the majority of results demonstrate that this intervention was not more effective than UC in managing glucometabolic and $\mathrm{CV}$ biomarkers in IHG. Future interventions should include additional support for the entire study duration however this has cost implications.
\end{abstract}

\section{Keywords}

Physical Activity, Cadence, Diabetic Health 


\section{Introduction}

Excess adiposity continues to present significant health and social problems [3]. Adults carrying excess adipose tissue are at higher risk of developing co-morbidities such as impaired glucose metabolism and cardiovascular disease (CVD) and have increased mortality rates [4]. As adiposity increases beyond a body mass index (BMI) of $25 \mathrm{~kg} / \mathrm{m}^{2}$, the risk of the development of type 2 diabetes mellitus (T2DM) also increases [5]. Many adults with hyperglycemia remain in an asymptomatic state for a prolonged period before T2DM diagnosis [6]. This intermediate hyperglycemia (IHG) includes impaired glucose tolerance (IGT) and/or impaired fasting glucose [1]. In addition to the impaired handling of glucose, IHG can increase oxidative stress, inflammation and endothelium dysfunction [6] [7] [8]. Approximately 86 million (37\% of the adult US population) meet the diagnostic criteria for IHG [9]. In the UK, around 7 million people are thought to have IHG [2] with estimates suggesting that 1 in 3 English adults have IHG [2] [10]. Like the majority of the population, less than half of this population report completing current PA guidelines [11]. It is well established that increasing PA in IHG is one method to reduce the risk of glucometabolic and CVD complications [2]. Moderate intensity exercise in particular is required to regulate hyperglycaemia by increasing GLUT-4 translocation to muscle tissue, enhancing uptake of glucose via PI-3K and MAP kinase pathways [12]. Similar effects are noted for the reduction of inflammation associated with diabetic progression [13]. Recent studies have calculated that $£ 1$ million per hour is spent from the National Health Service (NHS) budget on diabetes health care costs [14]. As such, preventing progression from IHG to T2DM using low cost, effective and sustainable methods is important for public health. Assisting individuals to achieve and maintain moderate intensity exercise remains problematic. Previous research has used heart rate monitors, self-report methods and accelerometers/ pedometers as tools, but many of these possess limitations from a user's perspective, but also a research reliability and validity perspective [15]. Using music with a pre-determined tempo to help stimulate and regulate cyclic PA intensity may ensure PA is regularly performed at the desired intensity [16].

The aims of this study were to examine the effects of a walking to music intervention to encourage a pace which achieves moderate intensity on glycemic control, oxidative stress, endothelial dysfunction and inflammatory markers in IHG.

\section{Methodology}

Following approval by Ulster University Research Ethics Committee and the Office for Research Ethics Northern Ireland (Approval number: 12/NI/0063), overweight and obese IHG adults who met the diagnostic criteria of a fasting plasma glucose between $5.6-6.9 \mathrm{mmol} / \mathrm{L}^{-1}$ or a two hour glucose concentration between $7.8-11.0 \mathrm{mmol} / \mathrm{L}^{-1}(n=42)$, were recruited from consultant led diabetic clinics and from a university within Northern Ireland (Figure 1). Exclusion 


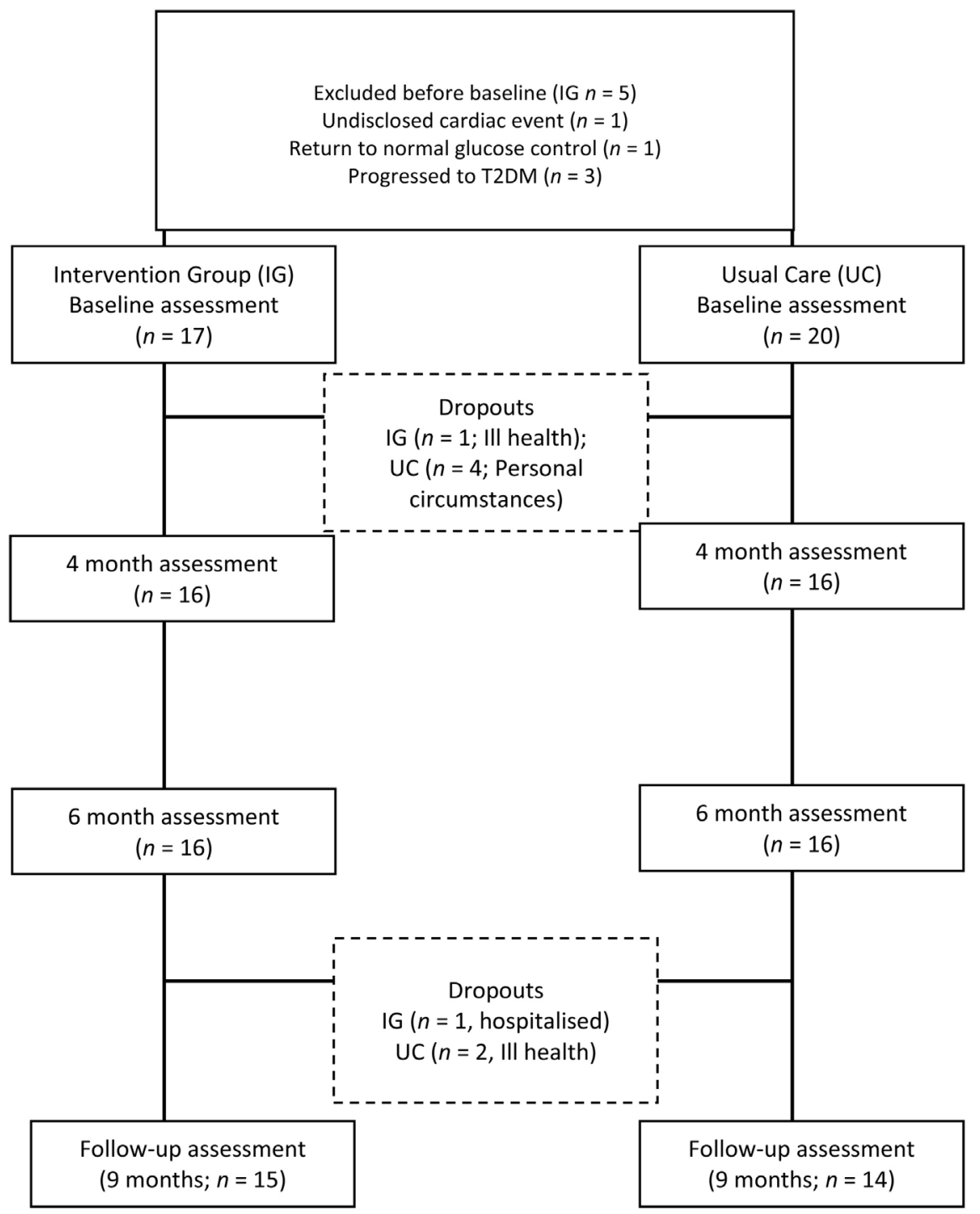

Figure 1. Flowchart of IG and UC participants from baseline to 9 months.

criteria for the study included those who had progressed to T2DM/those who had normoglycaemia, those under the age of 18 or over the age of 65 . Participants were also excluded if they were smokers, had a history of cardiovascular events, or were taking any medication which would interfere with the blood biomarkers assessed. Participants provided medical history details and informed consent before random assignment to an intervention group (IG) $(n=22)$ or usual care group (UC) $(n=20)$ (Figure 1). IG participants $(n=17)$ received an individualized 6 month walking program designed to increase moderate intensity walking to 30 minutes per day based on current PA guidelines [17]. Self-chosen music with a predetermined tempo was selected based upon height-related stride rate recommendations by Rowe et al. [18] to elicit moderate intensity exercise for each individual (50\% - 70\% maximum HR, 90 - 113 steps/min). Cadence (steps $/ \mathrm{min}^{-1}$ ) was recorded using the inbuilt MP3 accelerometer and analyzed via MATLAB (MATLAB, The Mathworks Inc, Natick, MA, 2014). IG received 
three physical activity consultations (PACS) during the 9 month intervention (0, 4 and 6 months) based on the work of Kirk and colleagues [19]. These were complimented by booster telephone sessions to revise goals, barriers to exercise and methods of achieving PA targets. UC participants $(n=20)$ received an information sheet detailing current PA guidelines and methods to achieve these on a weekly basis (this is the usual care those with IHG currently receive from the NHS). All measurements were completed at baseline, 4, 6 and 9 months (Figure 1).

\subsection{Physiological Testing}

Height $(\mathrm{cm})$ and body mass $(\mathrm{kg})$ were recorded and BMI $\left(\mathrm{kg} / \mathrm{m}^{2}\right)$ calculated [20]. Arterial blood pressure was measured in a supine position (Omron M5-1 BP monitor, Surrey, UK). DXA was used to measure whole and regional body composition and bone. Arterial stiffn-ness was assessed using a PulseTrace Pulse Contour Analysis (PCA) system (Micro Medical, UK). A 2D high resolution Sonoace Pico ultrasound system (GE Healthcare, Memphis TN) was used to assess endothelial function according to the methods of Harris et al. [21]. Physical fitness was measured via a sub-maximal graded exercise assessment using walking speeds determined by the ACSM metabolic equations [22]. Stages were 4 minutes in duration and consisted of three stages (12 minutes in total). Heart rate response and respiratory exchange values at each workload $\left(\mathrm{km} / \mathrm{hr}^{-1}\right)$ measured walking economy. PA was assessed via a self-administered International Physical Activity Questionnaire (IPAQ) short version [23]. IG intervention \% walking compliance was assessed by comparing the app-recorded walks (for volume and duration) to walking prescribed in each program. Intensity compliance (\%) was assessed by calculating the number of walks that, on average, were at/ above the predetermined target cadence. Both groups recorded PA in a logbook at each timepoint. At each timepoint, all participants completed a cadence walk around an elliptical track ( $\geq$ eight minutes) to ensure that the desired stride rate and moderate intensity exercise was completed. Fasted blood samples were assessed for the following metabolites. Glucometabolic status: fasting plasma glucose, oral glucose tolerance test (OGTT [24]), glycosylated hemoglobin $\left(\mathrm{HbA}_{1 \mathrm{C}}\right)$ and insulin; Lipoproteins. serum total cholesterol, high-density lipoprotein cholesterol (HDL-C), calculated low-density lipoprotein cholesterol (LDL-C) (Fried Wald et al. [25]) and serum triglyceride (TRIG). Inflammatory markers, tumor necrosis factor- $\alpha$ (TNF- $\alpha$ ), interleukin 6 (Il-6) and high-sensitivity $\mathrm{C}$ reactive protein (Hs-CRP); Oxidative stress markers. Lipid hydroperoxides (LOOHs) (FOX assay), lipid soluble antioxidants ( $\gamma$-tocopherol, $\alpha$-tocopherol; Retinol; Lycopene; $\beta$-carotene, $\alpha$-carotene) and ascorbyl free radical. Hematocrit and hemoglobin were also assessed via standard methods.

\subsection{Data Analysis}

Levene's test for equality of variances $(p>0.05)$ were performed to investigate 
homogeneity of variance. Independent-sample t-tests $(\mathrm{P})(p<0.05)$ were used to compare themeans of two groups' (IG vs. UC). A repeated measures GLM was utilized to assess the difference between variables with one between-subjects variable (two groups IG and UC), one within subject variable with two or more levels (e.g.4 time points: baseline, 4,6 and 9 months) and one continuous dependent variable (parameter measurement) $(p<0.05)$. All data is presented as mean value \pm standard deviation (SD) unless otherwise stated. Descriptive statistics (means or frequencies) were calculated where appropriate. All statistical analysis was completed using Microsoft Excel and SPSS version 22.0, for windows (SPSS, Chicago, IL).

\section{Results}

\subsection{Vascular Function and CVD Risk}

No statistically significant interaction (time $\times$ group) effects, main (time) effects or main (group) effects $(p>0.05)$ were observed for systolic and diastolic blood pressure $(\mathrm{mmHg})$ or resting heart rate (RHR; $\mathrm{BPM}^{-1}$ ) across assessment points (Table 1). No significant interaction (time $\times$ group) effect $\left(\mathrm{m} / \mathrm{sec}^{-1}\right)$ and no significant main (group) effect were found for SI $\left(\mathrm{m} / \mathrm{sec}^{-1}\right)$. SI $\left(\mathrm{m} / \mathrm{sec}^{-1}\right)$ displayed a significant main (time) effect $[\mathrm{F}(3,32)=2.938, p=0.048$, multivariate partial eta squared $=0.216]$. Post hoc comparisons found a statistical significance between SI baseline and 9-month values $(p=0.03)$. RI (\%) values displayed no significant interaction for time $\times$ group, time or group effect for RI(\%) values. $\mathrm{FMD}(\%)$ displayed a significant interaction effect (time $\times$ group) $[\mathrm{F}(3,32)=3.992, p=$ 0.016 , multivariate partial eta squared $=0.272]$. Post hoc analysis found a significant difference between IG and UC at 6 months $(\mathrm{t}(34)=2.74, p=0.10 ; 6.51 \% \pm$ $4.38 \%$ and $11.21 \% \pm 5.72 \%$ respectively). A significant increase was observed

Table 1. Summary of vascular function parameters.

\begin{tabular}{|c|c|c|c|c|c|c|c|c|c|c|c|c|c|c|c|c|}
\hline \multirow{3}{*}{ Parameter } & \multicolumn{8}{|c|}{ IG participants $(n=17)$} & \multicolumn{8}{|c|}{ UC participants $(n=19)$} \\
\hline & \multicolumn{2}{|c|}{ Baseline } & \multicolumn{2}{|c|}{4 months } & \multicolumn{2}{|c|}{6 months } & \multicolumn{2}{|c|}{9 months } & \multicolumn{2}{|c|}{ Baseline } & \multicolumn{2}{|c|}{4 months } & \multicolumn{2}{|c|}{6 months } & \multicolumn{2}{|c|}{9 months } \\
\hline & Mean & $\mathrm{SD}$ & Mean & $\mathrm{SD}$ & Mean & $\mathrm{SD}$ & Mean & SD & Mean & SD & Mean & $\mathrm{SD}$ & Mean & SD & Mean & SD \\
\hline \multicolumn{17}{|c|}{ Blood pressure } \\
\hline Systolic (mmHg) & 134.12 & 18.80 & 133.30 & 18.71 & 135.86 & 16.47 & 136.74 & 17.59 & 138.00 & 12.06 & 137.76 & 16.50 & 138.52 & 16.14 & 136.58 & 16.63 \\
\hline $\begin{array}{l}\text { Diastolic } \\
(\mathrm{mmHg})\end{array}$ & 81.20 & 11.81 & 78.86 & 9.82 & 82.73 & 13.57 & 81.62 & 10.55 & 79.60 & 8.60 & 78.21 & 7.32 & 77.56 & 10.59 & 78.50 & 9.30 \\
\hline RHR (bpm) & 66.90 & 10.26 & 64.49 & 9.39 & 66.23 & 13.93 & 64.73 & 10.16 & 67.96 & 8.61 & 69.20 & 9.43 & 68.52 & 8.80 & 65.60 & 9.17 \\
\hline \multicolumn{17}{|c|}{ Arterial Stiffness } \\
\hline $\mathrm{SI}\left(\mathrm{m} / \mathrm{sec}^{-1}\right)$ & 8.10 & 1.89 & 7.45 & 1.86 & 7.34 & 2.03 & 7.47 & 1.97 & 8.07 & 1.84 & 7.66 & 1.73 & 7.60 & 1.52 & 7.13 & 1.41 \\
\hline RI (\%) & 59.59 & 14.91 & 60.11 & 16.1 & 55.20 & 14.04 & 56.16 & 15.6 & 50.08 & 10.63 & 51.43 & 12.2 & 56.89 & 13.44 & 57.14 & 12.34 \\
\hline FMD (\%) & 9.50 & 5.59 & 7.24 & 3.15 & $6.51^{\dagger}$ & 4.38 & 7.81 & 3.82 & 9.66 & 4.75 & $7.40^{\wedge}$ & 2.85 & 11.21 & 5.72 & 8.47 & 3.81 \\
\hline
\end{tabular}

RHR $=$ resting heart rate, FMD = flow mediated dilation. A repeated measures GLM $(p<0.05)$ examined differences between groups across assessment points. ${ }^{\dagger}$ Denotes a significant difference between groups $(p<0.05)$. ${ }^{\wedge}$ Denotes a within group significant difference between given value and 6 month value $(p$ $<0.05)$. 
within UC FMD (\%) between 4 and 6 months $(p=0.017)$ with UC FMD percentage change increasing by $16.1 \%$ between these assessment points (as shown in Figure 2). A significant main (time) effect $[\mathrm{F}(3,32)=4.350, p=0.011$, multivariate partial eta squared $=0.290]$ was observed for FMD $(\%)$ between baseline and 4 months ( $p=0.015$ ). No significant main (group) effect was observed.

\subsection{Walking Compliance}

IG \% walking compliance from baseline to 4 months was $70.1 \% \pm 39.17 \%$ (range $9.0 \%$ to $158.4 \%$ ). This was followed by decreases to $43.4 \% \pm 56.1 \%$ (range $0 \%$ to $225 \%$ ) between 4 to 6 months and to $37.5 \% \pm 43.5 \%$ (range $0 \%$ to $125.3 \%$ ) at 9 months. Despite this, \% intensity compliance of the walks completed was $71.4 \%$, $79.5 \%$ and $73.3 \%$ was at least moderate intensity (achieving the predetermined target cadence) between baseline to four months, four to six months and six to nine months respectively. IPAQ data showed no significant interaction (group $\times$ time) effect, main (time) effect or main (group) effect in vigorous MET.min/week, moderate MET.min/week, walking MET.min/week or total PA MET.min/week $(p>0.05)$. At baseline participants were categorized as low active (50\% IG, 35\% UC), moderately active (30\% IG, 50\% UC) and highly active (20\% IG, 15\% UC). At 4 months participants were categorized as low active (45\% IG, $45 \%$ UC), moderately active (45\% IG, $40 \%$ UC) and highly active (10\% IG, $15 \%$ UC). At the end of the intervention (6 months) 55\% IG and 50\% UC participants were categorized as low active, $25 \%$ IG and $40 \%$ UC were categorized moderately active and $20 \%$ IG vs. $10 \%$ UC were categorized highly active. At follow-up (9 months) participants were categorized as low active (45\% IG, 45\% UC), moderately active (50\% IG, 45\% UC) and highly active (5\% IG, 10\% UC) (Table 2).

\subsection{Blood Biomarkers}

No significant interaction (time $\times$ group) and no main effect for group was

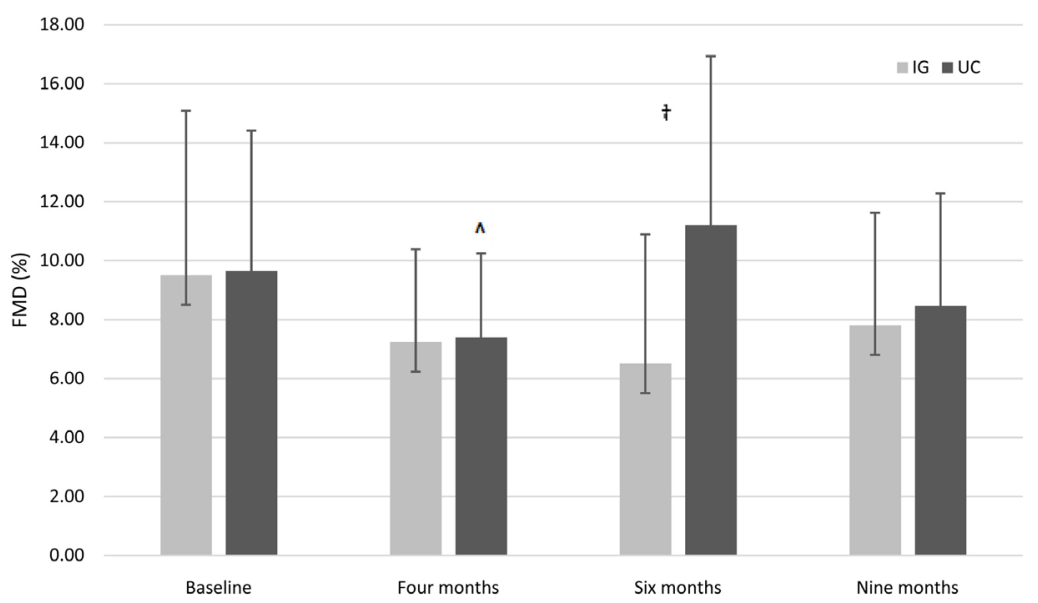

Figure 2. FMD (\%) across assessment points (IG $n=17$, UC $n=19)$ ) *A repeated measures GLM $(p<0.05)$ examined differences between groups across assessment points. ${ }^{\dagger}$ Denotes a significant difference between groups at 6 months $(p<0.05) .{ }^{\wedge}$ Denotes a within group significant difference between given value and 6 month value $(p<0.05)$ 
Table 2. IG and UC IPAQ continuous scores.

\begin{tabular}{|c|c|c|c|c|c|}
\hline \multirow[b]{2}{*}{ IPAQ DATA } & \multirow[b]{2}{*}{ Assessment } & \multicolumn{2}{|c|}{ IG participants $(n=17)$} & \multicolumn{2}{|c|}{ UC participants $(n=19)$} \\
\hline & & Median & $\begin{array}{l}\text { Interquartile } \\
\text { range }\end{array}$ & Median & $\begin{array}{c}\text { Interquartile } \\
\text { range }\end{array}$ \\
\hline \multirow{5}{*}{$\begin{array}{c}\text { Vigorous } \\
\text { MET.min/week }\end{array}$} & Baseline & 0 & 480 & 0 & 240 \\
\hline & 4 months & 0 & 0 & 0 & 0 \\
\hline & 6 months & 0 & 840 & 0 & 0 \\
\hline & 9 months & 0 & 0 & 0 & 0 \\
\hline & Baseline & 0 & 420 & 360 & 1248 \\
\hline \multirow{4}{*}{$\begin{array}{c}\text { Moderate } \\
\text { MET.min/week }\end{array}$} & 4 months & 200 & 400 & 240 & 760 \\
\hline & 6 months & 0 & 160 & 0 & 480 \\
\hline & 9 months & 0 & 160 & 0 & 520 \\
\hline & Baseline & 247.5 & 264 & 594 & 1204.5 \\
\hline \multirow{4}{*}{$\begin{array}{c}\text { Walking } \\
\text { MET.min/week }\end{array}$} & 4 months & 396 & 379.5 & 462 & 808.5 \\
\hline & 6 months & 346.5 & 660 & 462 & 693 \\
\hline & 9 months & 445.5 & 1089 & 495 & 825 \\
\hline & Baseline & 396 & 1811.4 & 1188 & 2826.5 \\
\hline $\begin{array}{c}\text { Total Physical } \\
\text { Activity }\end{array}$ & 4 months & 888 & 758 & 933 & 2535.5 \\
\hline \multirow[t]{2}{*}{ MET.min/ week } & 6 months & 792 & 1653 & 495 & 1459.5 \\
\hline & 9 months & 605.5 & 1752 & 990 & 1683.75 \\
\hline
\end{tabular}

Continuous IPAQ scores are presented as median and interquartile range. A repeated measures GLM found no statistically significant differences between groups across assessment points $(p>0.05)$.

detected for fasting plasma glucose. Non-significant lowering trends were observed for IG FPG $\left(\mathrm{mmol} / \mathrm{L}^{-1}\right)$ from baseline $\left(6.03 \pm 0.36 \mathrm{mmol} / \mathrm{L}^{-1}\right)$ to 4 months $\left(5.96 \pm 0.49 \mathrm{mmol} / \mathrm{L}^{-1}\right)$ and to 6 months $\left(5.95 \pm 0.41 \mathrm{mmol} / \mathrm{L}^{-1}\right)$. IG FPG was $5.95 \pm 0.41 \mathrm{mmol} / \mathrm{L}^{-1}$ at 6 months and $6.04 \pm 0.49 \mathrm{mmol} / \mathrm{L}^{-1}$ at 9 months. IG FPG at follow-up was lower than that recorded by UC participants $(6.04 \pm 0.49$ $\mathrm{mmol} / \mathrm{L}^{-1}$ vs. $6.10 \pm 0.48 \mathrm{mmol} / \mathrm{L}^{-1}$ respectively), this was anon-significant result $(p>0.05)$. No significant interaction (time $\times$ group) and no main effect for group was detected for two-hour glucose $\left(\mathrm{mmol} / \mathrm{L}^{-1}\right)$. There was a significant main (time) effect $[\mathrm{F}(3,32)=3.22, p=0.036]$ which displayed a large effect size [partial eta squared $=0.23$ ]. Post hoc analysis observed this significant effect for two-hour glucose $\left(\mathrm{mmol} / \mathrm{L}^{-1}\right)$ was found between baseline and 6 months $(p=$ 0.03 ) and baseline and 9 months $(p=0.03)$. Although no interaction (group $\times$ time) effect was found $(p>0.05)$ the greatest decrease in IG two-hour glucose $\left(\mathrm{mmol} / \mathrm{L}^{-1}\right)$ was $13.78 \%$ from baseline to 4 months $\left(7.29 \pm 2.27 \mathrm{mmol} / \mathrm{L}^{-1}\right.$ to 6.29 $\pm 1.96 \mathrm{mmol} / \mathrm{L}^{-1}$ respectively). UC two-hour glucose $\left(\mathrm{mmol} / \mathrm{L}^{-1}\right)$ decreased by $3.77 \%$ during the same period (baseline $8.00 \pm 2.72 \mathrm{mmol} / \mathrm{L}^{-1}$ to 4 months $7.70 \pm$ $\left.2.55 \mathrm{mmol} / \mathrm{L}^{-1} ; p>0.05\right)$ (Table 3 ).

\subsubsection{Glycosylated Hemoglobin ( $\mathrm{HbA}_{1 \mathrm{c}} ; \mathrm{mmol} / \mathrm{mol}^{-1}$ )}

No significant interaction (time $\times$ group) effect and no significant main (group) 
Table 3. Blood metabolites.

\begin{tabular}{|c|c|c|c|c|c|c|c|c|c|c|c|c|c|c|c|c|}
\hline \multirow{3}{*}{ Parameter } & \multicolumn{8}{|c|}{ IG participants $(\mathrm{n}=17)$} & \multicolumn{8}{|c|}{ UC participants $(\mathrm{n}=19)$} \\
\hline & \multicolumn{2}{|c|}{ Baseline } & \multicolumn{2}{|c|}{4 months } & \multicolumn{2}{|c|}{6 months } & \multicolumn{2}{|c|}{9 months } & \multicolumn{2}{|c|}{ Baseline } & \multicolumn{2}{|c|}{4 months } & \multicolumn{2}{|c|}{6 months } & \multicolumn{2}{|c|}{9 months } \\
\hline & Mean & SD & Mean & SD & Mean & SD & Mean & SD & Mean & SD & Mean & SD & Mean & SD & Mean & SD \\
\hline $\mathrm{FPG}\left(\mathrm{mmol} / \mathrm{L}^{-1}\right)$ & 6.03 & 0.36 & 5.96 & 0.49 & 5.95 & 0.41 & 6.04 & 0.49 & 6.20 & 0.47 & 6.21 & 0.83 & 6.18 & 0.57 & 6.10 & 0.48 \\
\hline $\begin{array}{l}2 \mathrm{hr} \text { gluc } \\
\left(\mathrm{mmol} / \mathrm{L}^{-1}\right)\end{array}$ & 7.29 & 2.27 & 6.29 & 1.96 & 6.52 & 1.68 & 6.67 & 1.98 & 8.00 & 2.72 & 7.70 & 2.55 & 6.69 & 2.71 & 6.35 & 1.85 \\
\hline $\begin{array}{l}\mathrm{HbA}_{1 \mathrm{c}} \\
\left(\mathrm{mmol} / \mathrm{mol}^{-1}\right)\end{array}$ & 38.1 & 5.2 & 37.7 & 4.9 & 36.7 & 3.9 & 36.7 & 4.3 & 39.4 & 5.5 & 37.9 & 5.8 & 38.9 & 5.7 & 38.5 & 3.6 \\
\hline Insulin $\left(\mathrm{pmol} / \mathrm{L}^{-1}\right)$ & 105.4 & 58.27 & 96.03 & 31.62 & 105.6 & 38.17 & 112.7 & 39.40 & 127.2 & 98.47 & 149.7 & 139.2 & 121.1 & 73.47 & 120.8 & 62.86 \\
\hline Haematocrit (\%) & 41.11 & 4.28 & 43.12 & 3.57 & 42.72 & 2.92 & 43.94 & 3.97 & 42.34 & 3.94 & 41.69 & 3.91 & 43.59 & 7.15 & 41.95 & 5.02 \\
\hline $\begin{array}{l}\text { Haemoglobin } \\
\left(\mathrm{g} / \mathrm{dL}^{-1}\right)\end{array}$ & 13.27 & 3.04 & 15.54 & 2.64 & 15.29 & 1.74 & 15.00 & 2.15 & 14.53 & 1.65 & 14.91 & 1.65 & 15.58 & 1.51 & 14.75 & 2.12 \\
\hline
\end{tabular}

FPG = fasting plasma glucose, $2 \mathrm{hr}$ gluc $=$ Two-hour glucose, $\mathrm{HbA}_{1 \mathrm{c}}=$ glycated haemoglobin. A repeated measures GLM found no statistically significant differences between groups across assessment points $(p>0.05)$.

effect was detected for $\mathrm{HbA}_{1 \mathrm{C}}\left(\mathrm{mmol} / \mathrm{mol}^{-1}\right)$. There was a significant main (time) effect found for $\mathrm{HbA}_{1 \mathrm{C}}\left(\mathrm{mmol} / \mathrm{mol}^{-1}\right.$; Table $3[\mathrm{~F}(3,32)=3.88, p=0.01]$ which displayed a large effect size [partial eta squared $=0.267$ ]. The significant main (time) effect was observed between baseline and 6 month values $\mathrm{HbA}_{1 \mathrm{C}}(p=$ $0.01)$. Although not significant between groups $(p>0.05)$ at the end of the intervention period (6 months) IG $\mathrm{HbA}_{1 \mathrm{C}}$ was $5.07 \%$ lower in comparison to UC values $\left(36.76 \pm 3.89 \mathrm{mmol} / \mathrm{mol}^{-1}\right.$ and $38.63 \pm 5.52 \mathrm{mmol} / \mathrm{mol}^{-1}$ respectively). At follow-up (9 months) a $4.00 \%$ difference was maintained (IG $36.81 \pm 4.29$ $\mathrm{mmol} / \mathrm{mol}^{-1}$ vs. UC $\left.38.28 \pm 4.37 \mathrm{mmol} / \mathrm{mol}^{-1} ; p>0.05\right)$.

\subsubsection{Insulin}

No significant interaction (time $\times$ group) effect with no main (time) effect or no significant main (group) effect were found for insulin ( $p>0.05$; Table 3). No significant interaction (time $\times$ group) effect with no main (time) effect or no main (group) effect $(p>0.05)$ were found for hematocrit (\%) and hemoglobin $\left(\mathrm{g} / \mathrm{dL}^{-1}\right)$.

\subsubsection{Lipoproteins (Table 4)}

CHOL $\left(\mathrm{mmol} / \mathrm{L}^{-1}\right)$ results displayed no significant interaction (time $\times$ group) and no significant main (group) effect. There was a significant main (time) effect $[\mathrm{F}(3,32)=5.37, p=0.00]$ which displayed a large effect size [partial eta squared $=$ 0.36 ] found for $\mathrm{CHOL}\left(\mathrm{mmol} / \mathrm{L}^{-1}\right)$. Post hoc comparisons found a significant increase between baseline $\left(4.74 \pm 1.02 \mathrm{mmol} / \mathrm{L}^{-1}\right)$ and 6 month $(5.06 \pm 1.07$ $\mathrm{mmol} / \mathrm{L}^{-1}$ ) CHOL $(p=0.004)$; (IG baseline and 6 month CHOL was $4.69 \pm 1.23$ $\mathrm{mmol} / \mathrm{L}^{-1}$ and $4.98 \pm 1.17 \mathrm{mmol} / \mathrm{L}^{-1}$ respectively, $\mathrm{UC}$ baseline and 6 month $\mathrm{CHOL}$ was $4.79 \pm 0.84 \mathrm{mmol} / \mathrm{L}^{-1} 5.12 \pm 1.01 \mathrm{mmol} / \mathrm{L}^{-1}$ respectively). HDL-C $\left(\mathrm{mmol} / \mathrm{L}^{-1}\right)$ displayed no significant interaction (time $\times$ group) effect with no main (time) effect or no significant main (group) effect $(p>0.05)$. LDL-C $\left(\mathrm{mmol} / \mathrm{L}^{-1}\right)$ displayed 
Table 4. Blood metabolites.

\begin{tabular}{|c|c|c|c|c|c|c|c|c|c|c|c|c|c|c|c|c|}
\hline \multirow{3}{*}{ Parameter } & \multicolumn{8}{|c|}{ IG participants $(n=17)$} & \multicolumn{8}{|c|}{ UC participants $(n=19)$} \\
\hline & \multicolumn{2}{|c|}{ Baseline } & \multicolumn{2}{|c|}{4 months } & \multicolumn{2}{|c|}{6 months } & \multicolumn{2}{|c|}{9 months } & \multicolumn{2}{|c|}{ Baseline } & \multicolumn{2}{|c|}{4 months } & \multicolumn{2}{|c|}{6 months } & \multicolumn{2}{|c|}{9 months } \\
\hline & Mean & SD & Mean & SD & Mean & SD & Mean & SD & Mean & SD & Mean & SD & Mean & SD & Mean & SD \\
\hline \multicolumn{17}{|c|}{ Lipoproteins } \\
\hline $\mathrm{CHOL}\left(\mathrm{mmol} / \mathrm{L}^{-1}\right)$ & 4.69 & 1.23 & 5.04 & 1.09 & 4.99 & 1.17 & 4.91 & 0.93 & 4.79 & 0.84 & 4.86 & 0.76 & 5.12 & 1.01 & 4.85 & 0.72 \\
\hline HDL-C $\left(\mathrm{mmol} / \mathrm{L}^{-1}\right)$ & 1.02 & 0.27 & 1.04 & 0.21 & 1.08 & 0.22 & 1.13 & 0.26 & 1.11 & 0.31 & 1.11 & 0.31 & 1.15 & 0.36 & 1.11 & 0.24 \\
\hline $\mathrm{LDL}-\mathrm{C}\left(\mathrm{mmol} / \mathrm{L}^{-1}\right)$ & 1.39 & 0.57 & 1.46 & 0.60 & 1.47 & 0.58 & 1.49 & 0.66 & 1.25 & 0.41 & 1.38 & 0.39 & 1.49 & 0.49 & 1.46 & 0.55 \\
\hline TRIG $\left(\mathrm{mmol} / \mathrm{L}^{-1}\right)$ & 3.05 & 1.15 & 3.34 & 1.01 & 3.24 & 1.07 & 3.11 & 0.90 & 3.11 & 0.77 & 3.12 & 0.70 & 3.30 & 0.89 & 3.08 & 0.78 \\
\hline \multicolumn{17}{|c|}{ Inflammatory markers } \\
\hline TNF- $\alpha\left(\mathrm{pg} / \mathrm{ml}^{-1}\right)$ & 1.35 & 1.62 & 9.61 & 27.60 & 7.69 & 19.19 & 1.09 & 1.71 & 0.75 & 0.68 & 1.81 & 4.51 & 2.00 & 3.27 & 0.74 & 0.63 \\
\hline IL-6 $\left(\mathrm{pg} / \mathrm{ml}^{-1}\right)$ & 29.14 & 45.29 & 38.36 & 47.25 & 13.82 & 36.28 & 33.60 & 44.16 & 27.66 & 38.97 & 11.57 & 16.09 & 14.11 & 28.52 & 37.31 & 36.45 \\
\hline Hs-CRP $\left(\mathrm{mg} / \mathrm{L}^{-1}\right)$ & 3.39 & 2.65 & 2.90 & 2.62 & 3.03 & 2.47 & 2.77 & 2.22 & 2.39 & 1.90 & 2.45 & 2.28 & 2.45 & 2.28 & 2.19 & 1.89 \\
\hline \multicolumn{17}{|c|}{ Oxidative stress markers } \\
\hline LOOHs $\left(\mathrm{mmol} / \mathrm{L}^{-1}\right)$ & 1.46 & 0.30 & 1.49 & 0.21 & 1.53 & 0.27 & 1.63 & 0.18 & 1.45 & 0.16 & 1.52 & 0.21 & 1.57 & 0.50 & 1.49 & 0.26 \\
\hline $\begin{array}{l}\gamma \text {-tocopherol } \\
\left(\mu \mathrm{mol} / \mathrm{L}^{-1}\right)\end{array}$ & $2.94^{¥}$ & 1.21 & $3.34^{*}$ & 1.97 & $3.31^{¥}$ & 1.87 & 5.64 & 2.39 & $3.99^{\wedge}$ & 2.18 & 3.39 & 1.99 & $2.66^{¥}$ & 0.88 & 4.30 & 1.82 \\
\hline $\begin{array}{l}\alpha \text {-tocopherol } \\
\left(\mu \mathrm{mol} / \mathrm{L}^{-1}\right)\end{array}$ & $25.32^{¥}$ & 12.59 & 32.87 & 15.24 & $33.14^{\dagger}$ & 15.66 & 44.50 & 18.07 & 34.34 & 21.39 & $32.03^{\wedge}$ & 15.18 & $21.52^{¥}$ & 8.59 & 33.95 & 14.03 \\
\hline Retinol $\left(\mu \mathrm{mol} / \mathrm{L}^{-1}\right)$ & $7.95^{¥}$ & 3.33 & 9.44 & 2.56 & 10.51 & 6.33 & $11.41^{\dagger}$ & 4.17 & 9.70 & 2.88 & 9.18 & 2.83 & 7.47 & 2.41 & 8.92 & 1.51 \\
\hline Lycopene $\left(\mu \mathrm{mol} / \mathrm{L}^{-1}\right)$ & $0.26^{¥}$ & 0.25 & $0.28^{¥}$ & 0.13 & $0.32^{\dagger ¥}$ & 0.16 & $0.01^{\dagger}$ & 0.04 & 0.22 & 0.26 & $0.33^{\wedge ¥}$ & 0.23 & 0.21 & 0.10 & 0.17 & 0.12 \\
\hline$\beta$-carotene $\left(\mu \mathrm{mol} / \mathrm{L}^{-1}\right)$ & 0.03 & 0.03 & 0.05 & 0.03 & 0.04 & 0.04 & 0.03 & 0.03 & 0.05 & 0.04 & 0.07 & 0.06 & 0.04 & 0.03 & 0.06 & 0.07 \\
\hline $\begin{array}{l}\alpha \text {-carotene } \\
\left(\mu \mathrm{mol} / \mathrm{L}^{-1}\right)\end{array}$ & 0.16 & 0.23 & 0.17 & 0.09 & 0.13 & 0.10 & 0.17 & 0.11 & 0.24 & 0.26 & 0.21 & 0.20 & 0.13 & 0.10 & 0.23 & 0.25 \\
\hline Ascorbyl & 4.69 & 1.23 & 5.04 & 1.09 & 4.99 & 1.17 & 4.91 & 0.93 & 4.79 & 0.84 & 4.86 & 0.76 & 5.12 & 1.01 & 4.85 & 0.72 \\
\hline
\end{tabular}

$\mathrm{CHOL}=$ Serum total cholesterol, HDL-C $=$ High-density lipoprotein cholesterol, LDL-C $=$ Low-density lipoprotein cholesterol, TRIG = Serum triglyceride, TNF- $\alpha=$ Tumor necrosis factor $-\alpha$, IL- $6=$ Interleukin, Hs-CRP = High-sensitivity C-reactive protein, LOOHs = Lipid hydroperoxides, Ascorbyl = Ascorbyl free radical. A repeated measures GLM $(p<0.05)$ examined differences between groups across assessment points. ${ }^{\dagger}$ Denotes a significant difference between groups $(p<0.05)$. ${ }^{\wedge}$ Denotes a within group significant difference between given value and 6 month value $(p<0.05)$. ${ }^{*}$ Denotes a within group significant difference between given value and 9 month value $(p<0.05)$.

no significant interaction (time $\times$ group) effect and no significant main (group) effect. There was a significant main (time) effect for $\operatorname{LDL}-\mathrm{C}[\mathrm{F}(3,32)=5.68, p=$ 0.00 , multivariate partial eta squared $=0.35$. Post hoc comparisons observed a significant increase between LDL-C $\left(\mathrm{mmol} / \mathrm{L}^{-1}\right)$ at baseline and 6 months $(p=$ 0.02). TRIG (mmol/ $\mathrm{L}^{-1}$ ) displayed no significant interaction (time $\mathrm{x}$ group) effect and no significant main (group) effect. There was a significant main (time) effect $[\mathrm{F}(3,32)=3.56, p=0.03$, multivariate partial eta squared $=0.25]$. Post hoc comparisons found a significant increase between TRIG $\left(\mathrm{mmol} / \mathrm{L}^{-1}\right)$ at baseline and 6 months $(p=0.04)$. Although no interaction (time $\times$ group) effect $(p>$ $0.05)$ was observed UC TRIG displayed an increasing trend from baseline to 6 months by $19.4 \%$ in contrast to IG $(5.8 \%)$. 


\subsubsection{Inflammatory Markers}

TNF- $\alpha\left(\mathrm{pg} / \mathrm{ml}^{-1}\right)$ displayed no significant interaction (time $\times$ group) effect and no significant main (group) effect. IL-6 $\left(\mathrm{pg} / \mathrm{ml}^{-1}\right)$ displayed no significant interaction (time $\times$ group) effect and no significant main (group) effect. There was a significant main (time) effect observed $[\mathrm{F}(3,32)=3.56, p=0.03$, multivariate partial eta squared $=0.25]$. Post hoc comparisons found a significant increase in IL-6 (PG/ml $\left.\mathrm{m}^{-1}\right)$ between 6 and 9 months $(p=0.03)$. Hs-CRP $\left(\mathrm{mg} / \mathrm{L}^{-1}\right)$ did not display a significant interaction (group $\times$ time) effect, with no significant main (time) effect and no significant main (group) effect $(p>0.05)$.

\subsubsection{Oxidative Stress Markers}

No significant interaction (time $\times$ group) effect was found for LOOHs $(p>0.05)$. No significant differences were observed for LOOHs $\left(\mathrm{mmol} / \mathrm{L}^{-1}\right)$ for main (time) effect and main (group) effect ( $p>0.05$ ). Lipid soluble antioxidants ( $\gamma$-tocopherol, $\alpha$-tocopherol, Retinol, Lycopene, $\beta$-carotene, $\alpha$-carotene $\gamma$-tocopherol $\left(\mu \mathrm{mol} / \mathrm{L}^{-1}\right)$ displayed a significant interaction (time $\times$ group) effect $[\mathrm{F}(3,32)=3.05, p=$ 0.03 , multivariate partial eta squared $=0.22]$. Post hoc comparisons found significant differences existed within groups. Statistical significance was found between IG baseline and 9 month $(p<0.01), 4$ and 9 month $(p<0.01)$ and between 6 and 9 month $(p=0.02) \gamma$-tocopherol $\left(\mu \mathrm{mol} / \mathrm{L}^{-1}\right)$ values. UC $\gamma$-tocopherol $\left(\mu \mathrm{mol} / \mathrm{L}^{-1}\right)$ values significantly differed between baseline and 6 months $(p<0.01)$ and between 6 and 9 months $(p<0.01)$. Following a main (time) effect $[\mathrm{F}(3,32)=$ 8.211, $p<0.01$, multivariate partial eta squared $=0.44]$, post hoc comparisons observed significant differences existed between $\gamma$-tocopherol $\left(\mu \mathrm{mol} / \mathrm{L}^{-1}\right)$ baseline and 9 month $(p=0.01), 4$ and 9 month $(p=0.010)$ and between 6 to 9 month $(p<0.01)$ values. See Table 4 for full antioxidant results. No significant interaction (time $\times$ group) effect, with no significant main (time) effect and no main (group) effect was observed for ascorbyl free radical $(p>0.05)$.

\subsection{Anthropometric Measurements}

Body mass $(\mathrm{kg})$ showed no statistically significant interaction (group $\times$ time) effect with no significant main (time) effect or no significant main (group) effect $(p>0.05)$. Although non-significant, IG body mass $(\mathrm{kg})$ displayed lowering trends from baseline to 4 months $(96.24 \pm 15.66 \mathrm{~kg}$ to $95.55 \pm 15.6 \mathrm{~kg})$ and from 4 months to 6 months $(95.55 \pm 15.6 \mathrm{~kg}$ to $94.89 \pm 16.36 \mathrm{~kg})$. IG body mass displayed similar results from 6 months to follow-up $(94.89 \pm 16.36 \mathrm{~kg}$ to $95.00 \pm$ $16.50 \mathrm{~kg} ; p>0.05)$. In contrast, although non-significant $(p>0.05) \mathrm{UC}$ body mass displayed an increasing trend from baseline to end of intervention (6 months; $92.61 \pm 18.53 \mathrm{~kg}$ to $93.23 \pm 19.31 \mathrm{~kg}$ ) (Table 1). No significant interaction (group $\times$ time) effect with no main (time) effect or no significant main (group) effect were found for BMI $\left(\mathrm{kg} / \mathrm{m}^{2} ; p>0.05\right)$. Although non-significant IG BMI $\left(\mathrm{kg} / \mathrm{m}^{2}\right)$ values lowered from baseline to 9 months $\left(34.19 \pm 6.33 \mathrm{~kg} / \mathrm{m}^{2}\right.$, $33.94 \pm 6.05 \mathrm{~kg} / \mathrm{m}^{2}, 33.88 \pm 6.65 \mathrm{~kg} / \mathrm{m}^{2}$ to $33.80 \pm 6.68 \mathrm{~kg} / \mathrm{m}^{2}$ respectively) while UC BMI $\left(\mathrm{kg} / \mathrm{m}^{2}\right)$ increased marginally throughout the intervention (0 - 6 months; 
$32.72 \pm 6.10 \mathrm{~kg} / \mathrm{m}^{2}$ to $33.0 \pm 5.70 \mathrm{~kg} / \mathrm{m}^{2}$ respectively; $\left.p>0.05\right)$.

\subsection{Dual-Energy X-Ray Absorptiometry (DXA)}

No significant interaction (time $\times$ group) effect, main (time) effect or main (group) effect was found in total body fat (\%) ( $p>0.05$; IG total body fat (\%) was $43.14 \pm$ $9.88 \mathrm{~kg}$ to $42.86 \pm 9.98 \mathrm{~kg}$ ( 0 - 9 months respectively); UC total body fat (\%) was $43.27 \pm 8.59 \mathrm{~kg}$ (baseline) and $43.45 \pm 43.43 \mathrm{~kg}$ (9 months). No statistically significant interaction (time $\times$ group) effect with no main (time) or main (group) effect was detected for regional fat (\%), gynoid fat (\%), total mass ( $\mathrm{kg})$, tissue mass $(\mathrm{g})$, fat mass $(\mathrm{g})$, lean mass $(\mathrm{g})$, fat free mass $(\mathrm{g})$ or $\mathrm{z}$ scores $(p>0.05)$. No statistically significant interaction (time $\times$ group) effect or main (time) effect was found for $\mathrm{t}$-scores. A significant main (group) effect $[\mathrm{F}(1)=7.766, p=0.009]$ was found. The effect size found was large [partial eta squared $=0.186$ ]. No statistically significant interaction (time $\times$ group) effect or main (group) effect was detected for BMC (g) $(p>0.05)$. A statistically significant main (time) effect was found between baseline and 6 month BMC (g) (IG 2829.50 $\pm 425.18 \mathrm{~g}$ to $2805 \pm$ $429.18 \mathrm{~g}$; UC $2703.42 \pm 518.32 \mathrm{~g}$ to $2692.53 \pm 519.00 \mathrm{~g}$ ) [F(2,33.0)=5.920, $p=$ 0.004]. The effect size was large [partial eta squared $=0.264$ ]. IG android fat $(\%)$ displayed lowering trends during the intervention (0 - 6 months; $53.75 \% \pm$ $9.15 \%$ to $52.85 \% \pm 8.69 \%$ ) and at follow-up (to $52.69 \% \pm 9.08 \%$ ) although this was not significant $(p>0.05)$. UC android fat $(\%)$ displayed a similar pattern from baseline to 9 months $(53.66 \% \pm 8.24 \%$ to $53.66 \% \pm 8.45 \%$ respectively; $p>$ 0.05 ). No statistically significant interaction (time $\times$ group) effect or no significant main (group) effect was detected for A/G ratio $(p>0.05)$. Following a repeated measures GLM a significant main (time) effect was detected for A/G ratio $[\mathrm{F}(35)=2, p=0.011]$. The effect size was large [partial eta squared $=0.240]$. Post hoc analysis displayed a statistically significant difference between baseline and 6 month A/G ratio scores $(p=0.08$; IG $1.21 \pm 0.24$ and $1.18 \pm 0.25$ respectively; $\mathrm{UC} 1.18 \pm 0.21$ and $1.16 \pm 0.21$ respectively).

\subsection{Physical Fitness Assessment}

A significant interaction (time $\times$ group) effect was found for heart rate response at given speeds throughout the study $[\mathrm{F}(3,32)=3.073, p=0.044$, multivariate partial eta squared $=0.224]$. Post hoc analysis found no significant difference between IG and UC at any assessment points $(p>0.05)$. Significant differences were found within UC for HR response at $4.2 \mathrm{~km} / \mathrm{hr}^{-1}$ between 4 and 9 months $(p=0.34)$, for HR response to $5.2 \mathrm{~km} / \mathrm{hr}^{-1}$ between 4 and 9 months $(p=0.016)$ and for HR response to $6.2 \mathrm{~km} / \mathrm{hr}^{-1}$ between 6 and 9 months $(p=0.008)$ (Table $5)$. No significant interaction (group $\times$ time) effect and no significant main (group) effect were observed for RPE. RPE displayed a significant main (time) effect. Post hoc comparisons found a statistical significance between baseline and 4 month $(p=0.024)$, baseline and 6 month $(p<0.001)$, baseline and 9 month $(p<0.001)$ and 4 and 6 month RPE values $(p=0.013)$. Although no significant interaction (time $\times$ group) effect was observed between groups $(p>$ 
Table 5. Physical fitness: sub-maximal treadmill walking assessment parameters.

\begin{tabular}{|c|c|c|c|c|c|c|c|c|c|c|c|c|c|}
\hline \multirow{3}{*}{ Parameter } & \multirow{3}{*}{ Assessment } & \multicolumn{4}{|c|}{$4.20 \mathrm{~km} / \mathrm{hr}^{-1}$} & \multicolumn{4}{|c|}{$5.20 \mathrm{~km} / \mathrm{hr}^{-1}$} & \multicolumn{4}{|c|}{$6.20 \mathrm{~km} / \mathrm{hr}^{-1}$} \\
\hline & & \multicolumn{2}{|c|}{$\mathrm{IG}(n=17)$} & \multicolumn{2}{|c|}{$\mathrm{UC}(n=19)$} & \multicolumn{2}{|c|}{$\mathrm{IG}(n=17)$} & \multicolumn{2}{|c|}{$\mathrm{UC}(n=19)$} & \multicolumn{2}{|c|}{ IG $(n=17)$} & \multicolumn{2}{|c|}{$\mathrm{UC}(n=19)$} \\
\hline & & Mean & SD & Mean & SD & Mean & SD & Mean & SD & Mean & SD & Mean & SD \\
\hline \multirow{4}{*}{$\begin{array}{l}\text { Heart rate } \\
\left(\mathrm{bpm}^{-1}\right)\end{array}$} & Baseline & 100.68 & 14.15 & 99.75 & 11.68 & 112.03 & 17.05 & 111.07 & 13.97 & 125.90 & 22.45 & 127.20 & 19.61 \\
\hline & 4 months & 98.66 & 12.75 & $100.94^{q}$ & 8.49 & 111.86 & 16.24 & $112.63^{¥}$ & 10.98 & 125.51 & 18.42 & 128.78 & 12.17 \\
\hline & 6 months & 97.11 & 14.09 & 100.35 & 8.19 & 108.81 & 17.38 & 113.09 & 10.41 & 126.04 & 20.35 & $131.16^{¥}$ & 15.14 \\
\hline & 9 months & 100.30 & 15.95 & 96.21 & 9.26 & 112.90 & 18.50 & 108.07 & 12.25 & 127.70 & 18.29 & 124.77 & 14.09 \\
\hline \multirow{4}{*}{$\begin{array}{c}\dot{V} \mathrm{O}_{2} \\
\left(\mathrm{ml} / \mathrm{kg} / \mathrm{min}^{-1}\right)\end{array}$} & Baseline & 10.18 & 2.64 & 10.75 & 2.75 & 11.91 & 2.62 & 12.48 & 3.03 & 14.15 & 2.57 & 15.01 & 4.00 \\
\hline & 4 months & 9.13 & 2.72 & 9.81 & 2.85 & 11.64 & 2.67 & 11.82 & 2.69 & 14.49 & 4.17 & 14.27 & 4.02 \\
\hline & 6 months & 9.69 & 2.51 & 11.07 & 1.73 & 12.43 & 2.61 & 13.10 & 2.05 & 15.59 & 2.20 & 15.69 & 2.40 \\
\hline & 9 months & 10.55 & 1.98 & 10.86 & 1.85 & 12.61 & 1.95 & 12.55 & 2.15 & 15.55 & 2.16 & 15.46 & 2.36 \\
\hline \multirow{4}{*}{$\begin{array}{c}\dot{\mathrm{V}} \mathrm{O}_{2} \\
\left(\mathrm{~L} / \mathrm{min}^{-1}\right)\end{array}$} & Baseline & 1.01 & 0.32 & 0.99 & 0.28 & 1.18 & 0.33 & 1.15 & 0.33 & 1.39 & 0.35 & 1.40 & 0.45 \\
\hline & 4 months & 0.92 & 0.32 & 0.92 & 0.32 & 1.13 & 0.36 & 1.09 & 0.34 & 1.40 & 0.49 & 1.32 & 0.49 \\
\hline & 6 months & 0.92 & 0.31 & 1.05 & 0.28 & 1.19 & 0.36 & 1.24 & 0.32 & 1.43 & 0.29 & 1.47 & 0.39 \\
\hline & 9 months & 1.01 & 0.28 & 0.99 & 0.27 & 1.23 & 0.32 & 1.18 & 0.30 & 1.48 & 0.38 & 1.44 & 0.36 \\
\hline \multirow{4}{*}{$\begin{array}{c}\dot{\mathrm{V}} \mathrm{CO}_{2} \\
\left(\mathrm{~L} / \mathrm{min}^{-1}\right)\end{array}$} & Baseline & 0.78 & 0.26 & 0.80 & 0.24 & 0.97 & 0.26 & 0.99 & 0.29 & 1.19 & 0.31 & 1.25 & 0.42 \\
\hline & 4 months & 0.76 & 0.25 & 0.74 & 0.23 & 1.04 & 0.30 & 0.96 & 0.25 & 1.35 & 0.38 & 1.22 & 0.36 \\
\hline & 6 months & 0.75 & 0.26 & 0.85 & 0.25 & 1.04 & 0.34 & 1.08 & 0.31 & 1.22 & 0.40 & 1.36 & 0.41 \\
\hline & 9 months & 0.83 & 0.23 & 0.83 & 0.23 & 1.09 & 0.32 & 0.97 & 0.34 & 1.29 & 0.32 & 1.34 & 0.36 \\
\hline \multirow{4}{*}{ METs } & Baseline & 2.90 & 0.76 & 3.04 & 0.77 & 3.38 & 0.75 & 3.54 & 0.87 & 4.04 & 0.69 & 4.26 & 1.14 \\
\hline & 4 months & 2.58 & 0.78 & 2.78 & 0.79 & 3.29 & 0.73 & 3.34 & 0.74 & 4.09 & 1.10 & 4.04 & 1.14 \\
\hline & 6 months & 2.78 & 0.71 & 3.17 & 0.48 & 3.60 & 0.72 & 3.75 & 0.57 & 4.48 & 0.59 & 4.49 & 0.69 \\
\hline & 9 months & 3.06 & 0.56 & 3.04 & 0.59 & 3.68 & 0.54 & 3.60 & 0.62 & 4.50 & 0.62 & 4.41 & 0.68 \\
\hline \multirow{4}{*}{ RPE } & Baseline & 10.18 & 1.24 & 8.95 & 1.78 & 13.35 & 2.29 & 11.82 & 3.12 & 18.04 & 3.44 & 15.23 & 5.10 \\
\hline & 4 months & 9.32 & 2.14 & 8.30 & 2.22 & 12.63 & 2.20 & 11.14 & 3.40 & 16.55 & 3.89 & 14.42 & 5.06 \\
\hline & 6 months & 8.99 & 1.25 & 8.12 & 1.29 & 11.88 & 1.85 & 10.52 & 2.63 & 15.50 & 2.74 & 13.70 & 3.69 \\
\hline & 9 months & 8.97 & 1.96 & 8.67 & 1.48 & 11.69 & 2.23 & 10.76 & 3.09 & 15.00 & 2.76 & 13.82 & 4.80 \\
\hline
\end{tabular}

METs = metabolic equivalents, RPE = rate of perceived exertion. Repeated measures GLM examined differences between groups across assessment points $(p<0.05) .{ }^{\mathrm{x}}$ Denotes a within group significant difference between given value and 9 month value $(p<0.05)$.

$0.05)$, at $5.2 \mathrm{~km} / \mathrm{hr}^{-1}$ and $6.2 \mathrm{~km} / \mathrm{hr}^{-1} \mathrm{RPE}$ displayed decreasing trends for both IG and UC across the study, IG RPE at $4.2 \mathrm{~km} / \mathrm{hr}^{-1}$ decreased from baseline to 9 months, while UC RPE at $4.2 \mathrm{~km} / \mathrm{hr}^{-1}$ decreased from baseline to 6 months before displaying an increasing trend between 6 and 9 months $(p>0.05)$.

\subsection{Discussion}

To the author's knowledge, this study is the first to demonstrate that walking to music with an individualized predetermined beat is a novel method to ensure PA guidelines for health can be achieved in a free-living environment for up to 9 months. The results have shown that although walking compliance (volume and duration) decreased over the study period from $70(+39) \%$ to $37.5(43.5) \%$; the majority of participants were able to attain their individualized moderate inten- 
sity target cadence between baseline to four months, four to six months and six to nine months (71.4\%, $79.5 \%$ and $73.3 \%$ respectively). In other words, over $70 \%$ of the physical activity which they did complete was at least moderate intensity or higher. Achieving moderate intensity PA in those with IHG is of particular importance as it is well recognized to reduce the risk of glucometabolic and CVD complications [2]. Previous research by Tudor-Locke and Rowe [16] has indicated that regulating intensity of PA in a free-living environment remains problematic. As such these results in terms of attaining moderate PA in an "at-risk" population who should be targeted for PA behaviour change interventions could be described as promising.

\subsubsection{Vascular Function and CVD Risk}

Despite these encouraging results in terms of attaining moderate intensity PA, one of themain findings of this study was that the current intervention was not effective in eliciting changes to the majority of glucometabolic and CVD biomarkers assessed. Some significant improvements were found in vascular function; however these should be interpreted with care. SI, which reflects the measurement of stiffness in the large arteries, displayed a significant main effect for time with values decreasing during the study (baseline to 6 months). RI values which reflect the vascular tone of the small arteries did not change throughout the study, however values positively decreased by $7.37 \%$ for the IG during the intervention in contrast to UC RI values which increased by $13.6 \%$ over the same period. FMD analysis demonstrated a significant interaction effect between groups at six months (IG, $6.51 \pm 4.38$ vs UC, $11.21 \pm 5.72$ ). Interestingly, significant increases were observed within UC FMD (\%) between 4 and 6 months $(7.40 \pm 2.85$ and $11.21 \pm 5.72$ respectively). Whilst FMD in IG displayed a lowering trend across all timepoints. Improvements in arterial stiffness and FMD are crucial in this population which are predisposed to increased CVD risk [26]. Research has highlighted the inverse relationship which exists between FMD and future cardiac event risk [27]. Indeed, a recent meta-analysis showed that for every $1 \%$ or 1 standard deviation decrease in FMD there is an associated increased risk of $8 \%$ or $22 \%$ respectively of suffering a future CVD event [28]. Previous research has shown the reliability of FMD is a particularly intricate technique [29] and as such it is felt an important methodological concern which should be published in relation to the current population. FMD technique relies on skilled acquisition and interpretation of results [30] and as such requires replication in a larger cohort to confirm these findings. Several measurement issues are possible when assessing FMD in obese IHG populations, which require consideration. These include sizing issues with cuff and plinth, inability to lie supine for required sampling and larger quantities of adipose tissue introducing difficulty in isolating the brachial artery for analysis. Previous research has shown that improvements in SI and FMD may be the result of reduced circulating lipids and increased NO availability [26]. This study observed favorable improvements in FMD in the absence of improvements in lipoproteins, adipose tissue mass and 
Hs-CRP $\left(\mathrm{mg} / \mathrm{L}^{-1}\right)$. Reductions in Hs-CRP are normally associated with increased nitric oxide (NO) expression which leads to enhanced NO bioavailability and improved FMD [31]. Although Hs-CRP did not change significantly, the observed decreasing trends may have been sufficient to have impacted on FMD. However, it is important to consider the methodological issues encountered and note that statistical power of this study was based on two-hour glucose rather than FMD.

\subsubsection{Glycemic Control}

A main finding of this study was that the current intervention was not effective in significantly improving glycemic control (FPG and 2-hour glucose). IG 2-hour glucose decreased by $13.8 \%$ from baseline to 4 months while UC 2-hour glucose decreased by $3.8 \%$ during the same period. This is contrary to the PREPARE program by Yates and colleagues [32] who employed a structured educational process for IHG participants based on the DESMOND program [33] and did find significant differences in 2-hour glucose. It should be noted that previous studies which found significances in glucose metabolites were delivered by large multi-disciplinary teams beyond the scope of the current research [34] [35]. This again raises the question of whether behavioral support in the current study should have been extended and increased beyond the 6 month initial intervention; however this has obvious time and fiscal implications. $\mathrm{HbA}_{1 \mathrm{C}}$ identifies average plasma glucose over a period of approximately 8 to 12 weeks [24]. Although no significance was found for $\mathrm{HbA}_{1 \mathrm{C}}$ between groups, a significant main effect for time was displayed. Interestingly, although non-significant between groups at the end of the intervention (6 months) IG $\mathrm{HbA}_{1 \mathrm{C}}$ displayed a $5.07 \%$ lower result in contrast to UC values. Furthermore, at follow-up ( 9 months) a $4 \%$ difference was maintained (IG $36.81 \pm 4.29 \mathrm{mmol} / \mathrm{mol}^{-1}$ vs. UC $38.28 \pm 4.37$ $\left.\mathrm{mmol} / \mathrm{mol}^{-1}\right)$. The UK prospective diabetes study group [36] has previously shown that reducing $\mathrm{HbA}_{1 \mathrm{C}}$ by $11 \mathrm{mmol} / \mathrm{mol}^{-1}$ (or $1 \%$ ) reduces the risk of individuals with T2DM developing diabetic associated complications. A $1 \% \mathrm{Hb}_{1 \mathrm{C}}$ reduction decreases the risk of mortality, myocardial infarction and microvascular complications by $14 \%, 21 \%$, and $37 \%$ respectively [36]. Therefore, improving glycemic control via a reduction in $\mathrm{HbA}_{1 \mathrm{C}}$ is advantageous in IHG and has been achieved to some degree in this study.

\subsubsection{Anthropometry}

Previous IHG and T2DM interventions have focused on decreasing body mass in overweight individuals [34]. However, it is now clear that improved glucose metabolism and CVD risk reduction can be achieved in the absence of weight loss [37]. This study did not find a significant difference in body fat percentage. IG android fat (\%) decreased from $53.8 \% \pm 9.2 \%$ (baseline) to $52.9 \% \pm 8.7 \%$ at the end of the intervention (6 months), decreases which were maintained at follow up and within the UC group. Centrally located android (\%) and gynoid (\%) has been associated with increased risk of CVD [38] therefore these results sug- 
gest that IG android and gynoid fat (\%) displayed favorable improvements.

\subsubsection{Inflammatory and Oxidative Stress Markers}

IL- 6 and TNF- $\alpha$ are cytokines associated with skeletal muscle inflammation and feature in obesity related insulin sensitivity [39]. These increases cause the chronically stimulation of the NF- $\mathrm{kB}$ pathways which increases insulin resistance and $\beta$-cell dysfunction [40]. In turn, increased oxidative stress can be caused by ROS directly damaging cellular macromolecules [40]. In the current study a significant main (time) effect was observed for IL- $6\left(\mathrm{pg} / \mathrm{ml}^{-1}\right)$ from 6 to 9 months. While IL-6 $\left(\mathrm{pg} / \mathrm{ml}^{-1}\right)$ displayed a significant increase over time between 6 months and 9 months, it is worth noting follow-up concentrations remained lower for IG participants suggesting a reduced inflammatory response. Harris et al. [41] found that IL-6 ( $\left.\mathrm{pg} / \mathrm{ml}^{-1}\right)$ production during acute exercise is dependent on the intensity of activity and has the ability to directly inhibit TNF- $\alpha$. In contrast to moderate $\left(50 \% \mathrm{VO}_{2 \max }\right)$ and vigorous itensity $\left(75 \% \mathrm{VO}_{2 \max }\right)$ activity $(p<0.05)$, one hour post low intensity activity $\left(25 \% \mathrm{VO}_{2 \max }\right)$ elicited no significant effects on IL-6 ( $\left.\mathrm{pg} / \mathrm{ml}^{-1}\right)$ production [21]. In the current study no significance was observed for TNF- $\alpha\left(\mathrm{pg} / \mathrm{ml}^{-1}\right)$ however, IG TNF- $\alpha\left(\mathrm{pg} / \mathrm{ml}^{-1}\right)$ displayed lowering trends from baseline to 4 months and recorded its lowest value at 9 months. In contrast UC TNF- $\alpha\left(\mathrm{pg} / \mathrm{ml}^{-1}\right)$ values displayed non-significant increasing trends throughout the intervention and from baseline to follow-up (0 - 6 months and 0 - 9 months). This may be due to the observed lack of reduction in fat mass parameters as has been found by Katsuki et al. [42]. Ascorbyl free radical and LOOHs are indicators of oxidative stress [43]. In the current study no statistical significance was found between groups for either of these markers. IG LOOHs increased from baseline to 9 months at each time point. Interestingly, a significant interaction effect was observed for numerous lipid soluble antioxidants ( $\gamma$-tocopherol and $\alpha$-tocopherol, retinol and lycopene. IG $\gamma$-tocopherol and $\alpha$-tocopherol displayed similar patterns, increasing continually from baseline to follow-up. In contrast UC values decreased throughout the intervention (0 - 6 months). Similarly, IG retinol increased from baseline to 9 months (43.61\%) across all assessment points while UC retinol decreased throughout the study. IG lycopene displayed favorable increases from baseline to 6 months and in contrast UC lycopene decreased throughout the study. It may be suggested that the increased antioxidant defense observed above may have been sufficient to control any oxidative stress caused by increased moderate intensity activity. Dietary manipulation did not form part of the study but it is possible that the increase in PA led to changes in antioxidant intake as observed in previous research [44].

\section{Conclusion}

To the authors' knowledge, this is the first study to demonstrate that using music with a predetermined tempo can assist overweight adults with IHG to regulate their walking cadence to moderate intensity in a free-living environment. Furthermore, we have demonstrated that it is feasible to complete a 6 month 
walking to music intervention in an "at risk" population assessing a comprehensive range of physiological measures and biomarkers at 4 time points (including 9 month follow up). This study showed the novel walking intervention and individualized PACs were not more effective than standard NHS care in managing glucose metabolism overweight adults with IHG. This study did achieve significant improvements in FMD (mainly in the UC group); however, as discussed previously, these findings should be accepted with caution. A number of significant metabolic health improvements were observed over time but not between groups. It is suggested that future research examines the effect of walking to music with these parameters as primary outcome measures. It is strongly recommended that behavior change support embedded within PA interventions should be maintained for as long as feasibly and financially possible to determine whether effects on similar parameters may be improved.

\section{Funding}

This work was supported by a Department of Education (Northern Ireland) grant as part of the $\mathrm{PhD}$ project of Maria Faulkner.

\section{Conflicts of Interest}

The authors declare no conflicts of interest regarding the publication of this paper.

\section{References}

[1] Tarasova, V.D., Caballero, J.A., Turner, P. and Inzucchi, S.E. (2014) Speaking to Patients about Diabetes Risk: Is Terminology Important? Clinical Diabetes, 32, 90-95. https://doi.org/10.2337/diaclin.32.2.90

[2] Diabetes, U.K. (2015) Diabetes: Facts and Stats. https://www.diabetes.org.uk/Documents/About\%20Us/Statistics/Diabetes-key-stats -guidelines-April2014.pdf

[3] World Health Organisation (2015) Overweight and Obesity: Fact Sheet. http://www.who.int/mediacentre/factsheets/fs311/en/

[4] Tobias, D.K., Pan, A., Jackson, C.L., O’Reilly, E.J., Ding, E.L., Willett, W.C., Manson, J.E. and Hu, F.B. (2014) Body-Mass Index and Mortality among Adults with Incident Type 2 Diabetes. The New England Journal of Medicine, 370, 233-244. https://doi.org/10.1056/NEJMoa1304501

[5] Yang, L. and Colditz, G.A. (2015) Prevalence of Overweight and Obesity in the United States 2007-2012. JAMA Internal Medicine, 175, 1412-1413. https://doi.org/10.1001/jamainternmed.2015.2405

[6] Bansal, N. (2015) Prediabetes Diagnosis and Treatment: A Review. World Journal of Diabetes, 6, 296-303. http://dx.doi.org/10.4239/wjd.v6.i2.296

[7] McNeilly, A.M., Davison, G.W., Murphy, M.H., Nadeem, N., Trinick, T., Duly, E., Novials, A. and McEneny, J. (2011) Effect of $\alpha$-Lipoic Acid and EXERCISE training on Cardiovascular Disease Risk in Obesity with Impaired Glucose Tolerance. Lipids in Health and Disease, 10, Article No. 217. https://doi.org/10.1186/1476-511X-10-217 
[8] Shah, A.D., Vittinghoff, E., Kandula, N.R., Srivastava, S. and Kanaya, A.M. (2015) Correlates of Prediabetes and Type II Diabetes in US South Asians: Findings from the Mediators of Atherosclerosis in South Asians Living in America (MASALA) Study. Annals of Epidemiology, 25, 77-83.

https://doi.org/10.1016/j.annepidem.2014.10.013

[9] Centre for Disease Control and Prevention (2014) Estimates of Diabetes and Its Burden in the United States. United States Department of Health and Human Services, Washington DC.

https://www.cdc.gov/diabetes/data/statistics/statistics-report.html

[10] Mainous, A.G., Tanner, R.J., Baker, R., Zayas, C.E. and Harle, C.A. (2014) Prevalence of Prediabetes in England from 2003 to 2011: Population-Based, Cross-Sectional Study. BMJ Open, 4, e005002. https://doi.org/10.1136/bmjopen-2014-005002

[11] Gopalan, A., Lorincz, I.S., Wirtalla, C., Marcus, S.C. and Long, J.A. (2015) Awareness of Prediabetes and Engagement in Diabetes Risk-Reducing Behaviors. American Journal of Preventive Medicine, 49, 512-519. https://doi.org/10.1016/j.amepre.2015.03.007

[12] Ruiz-Alcaraz, A.J., Lipina, C., Petrie, J.R., Murphy, M.J., Morris, A.D., Sutherland, C. and Cuthbertson, D.J. (2013) Obesity-Induced Insulin Resistance in Human Skeletal Muscle Is Characterised by Defective Activation of p42/p44 MAP Kinase. PLOS ONE, 8, e56928. https://doi.org/10.1371/journal.pone.0056928

[13] Teixeira-Lemos, E., Nunes, S., Teixeira, F. and Reis, F. (2011) Regular Physical Exercise Training Assists in Preventing Type 2 Diabetes Development: Focus on Its Antioxidant and Anti-Inflammatory Properties. Cardiovascular Diabetology, 28, Article No. 12. https://doi.org/10.1186/1475-2840-10-12

[14] Hex, N., Bartlett, C., Wright, D., Taylor, M. and Varley, D. (2012) Estimating the Current and Future Costs of Type 1 and Type 2 Diabetes in the UK, Including Direct Health Costs and Indirect Societal and Productivity Costs. Diabetic Medicine, 29, 855-862. https://doi.org/10.1111/j.1464-5491.2012.03698.x

[15] Tudor-Locke, C. and Bassett, D. (2004) How Many Steps/Day Are Enough? Sports Medicine, 34, 1-8. https://doi.org/10.2165/00007256-200434010-00001

[16] Tudor-Locke, C. and Rowe, D.A. (2012) Using Cadence to Study Free-Living Ambulatory Behaviour. Sports Med, 42, 381-398. https://doi.org/10.2165/11599170-000000000-00000

[17] Physical Activity Guidelines: UK Chief Medical Officers' Report. A Report from the Chief Medical Officers in the UK on the Amount and Type of Physical Activity People Should Be Doing to Improve Their Health (2019). https://www.gov.uk/government/publications/physical-activity-guidelines-uk-chiefmedical-officers-report

[18] Rowe, D.A., Welk, G.J., Heil, D.P., Mahar, M.T., Kemble, C.D., Calabró, M.A. and Camenisch, K. (2011) Stride Rate Recommendations for Moderate-Intensity Walking. Medicine \& Science in Sports \& Exercise, 43, 312-318. https://doi.org/10.1249/MSS.0b013e3181e9d99a

[19] Kirk, A., Barnett, J., Leese, G. and Mutrie, N. (2009) A Randomized Trial Investigating the 12-Month Changes in Physical Activity and Health Outcomes following a Physical Activity Consultation Delivered by a Person or in Written form in Type 2 Diabetes: Time2Act. Diabetic Medicine, 26, 293-301.

https://doi.org/10.1111/j.1464-5491.2009.02675.x

[20] Scholfield, W.N., Scholfield, C. and James, W.P.T. (1985) Human Nutrition. Human Nutrition: Clinical Nutrition, 39, 1-96.

[21] Harris, R.A. (2010) FMD, Reproducibility, and Acute Exercise in the Obese: Are the 
Results Confounded? European Journal of Applied Physiology, 109, 357-358. https://doi.org/10.1007/s00421-009-1343-8

[22] Glass, S. and Dwyer, G.B. (2007) ACSM's Metabolic Calculations Handbook (American College of Sports Medicine). Lippincott Williams \& Wilkins, Baltimore.

[23] Craig, C., Marshall, A., Sjöström, M., Bauman, A., Booth, M., Ainsworth, B., Pratt, M., Ekelund, U., Yngve, A., Sallis, J., IPAQ Consensus Group and the IPAQ Reliability and Validity Study Group (2003) International Physical Activity Questionnaire (IPAQ): 12-Country Reliability and Validity. Medicine \& Science in Sports \& Exercise, 35, 1381-1395. https://doi.org/10.1249/01.MSS.0000078924.61453.FB

[24] World Health Organisation (2006) Definition and Diagnosis of Diabetes Mellitus and Intermediate Hyperglycemia: Report of a WHO/IDF Consultation.

https://www.who.int/diabetes/publications/Definition\%20and\%20diagnosis\%20of\% 20diabetes new.pdf

[25] Friedwald, W.T., Levy, R.I. and Fredrickson, D.S. (1972) Estimation of the Concentration of Low-Density Lipoprotein Cholesterol in Plasma, without the Use of Preparative Ultracentrifuge. Clinical Chemistry, 18, 449-502.

https://doi.org/10.1093/clinchem/18.6.499

[26] Ziccardi, P., Nappo, F., Giugliano, G., Esposito, K., Marfella, R., Cioffi, M., D’Andrea, F., Molinari, A.M. and Giugliano, D. (2002) Reduction of Inflammatory Cytokine Concentrations and Improvement of Endothelial Functions in Obese Women after Weight Loss over One Year. Circulation, 105, 804-809. https://doi.org/10.1161/hc0702.104279

[27] Ras, R.T., Streppel, M.T., Draijer, R. and Zock, P.L. (2013) Flow-Mediated Dilation and Cardiovascular Risk Prediction: A Systematic Review with Meta-Analysis. International Journal of Cardiology, 168, 344-351. https://doi.org/10.1016/j.ijcard.2012.09.047

[28] Inaba, Y., Chen, J.A. and Bergmann, S.R. (2010) Prediction of Future Cardiovascular Outcomes by Flow-Mediated Vasodilatation of Brachial Artery: A Meta-Analysis. The International Journal of Cardiovascular Imaging, 26, 631-640. https://doi.org/10.1007/s10554-010-9616-1

[29] Onkelinx, S., Cornelissen, V., Goetschalckx, K., Thomaes, T., Verhamme, P. and Vanhees, L. (2012) Reproducibility of Different Methods to Measure the Endothelial Function. Vascular Medicine, 17, 79-84. https://doi.org/10.1177/1358863X12436708

[30] Thijssen, D.H., Black, M.A., Pyke, K.E., Padilla, J., Atkinson, G., Harris, R.A., Parker, B., Widlansky, M.E., Tschakovsky, M.E. and Green, D.J. (2011) Assessment of Flow-Mediated Dilation in Humans: A Methodological and Physiological Guideline. American Journal of Physiology-Heart and Circulatory Physiology, 300, H2H12. https://doi.org/10.1152/ajpheart.00471.2010

[31] Mozos, I., Malainer, C., Horbańczuk, J., Gug, C., Stoiam, D., Tudor Luca, C. and Atanasov, A.G. (2017) Inflammatory Markers for Arterial Stiffness in Cardiovascular Diseases. Frontiers in Immunology, 8, 1058. https://doi.org/10.3389/fimmu.2017.01058

[32] Yates, T., Davies, M., Gorely, T., Bull, F. and Khunti, K. (2009) Effectiveness of a Pragmatic Education Program Designed to Promote Walking Activity in Individuals with Impaired Glucose Tolerance. Diabetes Care, 32, 1404-1410. https://doi.org/10.2337/dc09-0130

[33] Skinner, T.C., Carey, M.E., Cradock, S., Daly, H., Davies, M.J., Doherty, Y., Heller, S., Khunti, K. and Oliver, L. (2006) Diabetes Education and Self-Management for 
Ongoing and Newly Diagnosed (DESMON); Process Modelling of Pilot Study. Patient Education and Counseling, 64, 369-377. https://doi.org/10.1016/j.pec.2006.04.007

[34] Tuomilehto, J., Lindström, J., Eriksson, J.G., Valle, T.T., Hämäläinen, H., IlanneParikka, P., Keinänen-Kiukaanniemi, S., Laakso, M., Louherantan, A. and Rastas, M. (2001) Prevention of Type 2 Diabetes Mellitus by Changes in Lifestyle among Subjects with Impaired Glucose Tolerance. The New England Journal of Medicine, 344, 1343-1350. https://doi.org/10.1056/NEJM200105033441801

[35] Yates, T., Davies, M., Gorely, T., Bull, F. and Khunti, K. (2008) Rationale, Design and Baseline Data from the Pre-Diabetes Risk Education and Physical Activity Recommendation and Encouragement (PREPARE) Program Study: A Randomized Controlled Trial. Patient Education and Counseling, 73, 264-271.

https://doi.org/10.1016/j.pec.2008.06.010

[36] Stratton, I.M., Adler, A.I., Neil, H.A., Matthews, D.R., Manley, S.E., Cull, C.A., Hadden, D., Turner, R.C. and Holman, R.R. (2000) Association of Glycaemia with Macrovascular and Microvascular Complications of Type 2 Diabetes (UKPDS 35): Prospective Observational Study. BMJ, 321, 405-412.

https://doi.org/10.1136/bmj.321.7258.405

[37] Ponsonby, A., Sun, C., Ukoumunne, O.C., Pezic, A., Venn, A., Shaw, J.E., Dunstan, D.W., Barr, E.L., Blair, S.N. and Cochrane, J. (2011) Objectively Measured Physical Activity and the Subsequent Risk of Incident Dysglycemia: The Australian Diabetes, Obesity and Lifestyle Study (AusDiab). Diabetes Care, 34, 1497-1502.

https://doi.org/10.2337/dc10-2386

[38] Hariri, A.A., Oliver, N.S., Johnston, D.G., Stevenson, J.C. and Godsland, I.F. (2013) Adiposity Measurements by BMI, Skinfolds and Dual Energy X-Ray Absorptiometry in Relation to Risk Markers for Cardiovascular Disease and Diabetes in Adult Males. Disease Markers, 35, Article ID: 763907. https://doi.org/10.1155/2013/763907

[39] Hardman, A.E., Stensel, D.J. (2009) Physical Activity and Health: The Evidence Explained. Routledge, London. https://doi.org/10.4324/9780203890714

[40] Evans, J.L., Goldfine, I.D., Maddux, B.A. and Grodsky, G.M. (2002) Oxidative Stress and Stress-Activated Signaling Pathways: A Unifying Hypothesis of Type 2 Diabetes. Endocrine Reviews, 23, 599-622. https://doi.org/10.1210/er.2001-0039

[41] Harris, R.A., Padilla, J., Hanlon, K.P., Rink, L.D. and Wallace, J.P. (2008) The Flow-Mediated Dilation Response to Acute Exercise in Overweight Active and Inactive Men. Obesity, 16, 578-584. https://doi.org/10.1038/oby.2007.87

[42] Katsuki, A., Sumida, Y., Murashima, S., Murata, K., Takarada, Y., Ito, K., Fujii, M., Tsuchihashi, K., Goto, H. and Nakatani, K. (1998) Serum Levels of Tumor Necrosis Factor- $\alpha$ Are Increased in Obese Patients with Noninsulin-Dependent Diabetes Mellitus 1. The Journal of Clinical Endocrinology \& Metabolism, 83, 859-862. https://doi.org/10.1210/jcem.83.3.4618

[43] Peitri, S., Segin, J.R., d'Arbigny, P.D. and Culcasi, M. (1994) Ascorbyl Free Radical: A Noninvasive Marker of Oxidative Stress in Human Open-Heart Surgery. Free Radical Biology and Medicine, 16, 523-528. https://doi.org/10.1016/0891-5849(94)90131-7

[44] Leon, A.S., Conrad, J., Hunninghake, D.B. and Serfass, R. (1979) Effects of a Vigorous Walking Program on Body Composition, and Carbohydrate and Lipid Metabolism of Obese Young Men. The American Journal of Clinical Nutrition, 32, 17761787. https://doi.org/10.1093/ajcn/32.9.1776 